\title{
En torno a la traducción de Cantos quechuas de José María Arguedas
}

\author{
César G. Saldaña Fernández y Mario Mejia huamán \\ Universidad Ricardo Palma
}

\section{Presentación}

Aunándonos al homenaje con motivo del trigésimo aniversario de la muerte del ilustre escritor, antropólogo y etnólogo mestizo José María Arguedas, presentamos a continuación un comentario crítico al pequeño libro Cantos y cuentos quechuas, publicado como parte de la serie Munilibros $\mathrm{N}^{\circ} 12$, en diciembre de 1986.' Y, como quechuahablantes, al iniciar nuestra exposición, reconocemos muy profundamene la obra que le tocó cumplir a José Maria Arguedas en la apertura del mundo campesino quechua hacia el mundo campesino citadino occidental y viceversa.

Tal vez José María Arguedas haya exagerado el valor mítico, místico y mágico del mundo quechua; pero, como la literatura no es una fotografia exacta del mundo, sino como una pintura reafista, dependen de artista tanto la selección como el ángulo pintađฮ̄e Puccinelli Converso»

El autor aportó mucho a la cultura, y trascendió positivamente al mundo andino y occidental como un misionero enviado a dar un mensaje, y no estuvo exento de virtudes, apasionamiento y limitaciones humanas, que ocasionaron incluso, la crítica aparentemente despiadada de Mario Vargas Llosa en La utopia arcaica. $^{2}$

I Esta obra fue publicada por vez primera en Lima, en 1939, por Ediciones Club del Libro Peruano. El autor fue quechuahablante de nacimiento muy conocido en el campo de la etnologia, la antropologia y la literatura; entre sus principales obras podemos señalar: Yawar fiesta, Los rios profundos. El zorro de arriba y el zorro de abajo y Todas las sangres.

2 Vargas Llosa, Mario. La utopia arcaica: José Maria Arguedas y las ficciones del indigenismo. México: Fondo de Cultura Económica. México, 1996, p. 359. 
Por aquella fecha de su publicación, por la Municipalidad de Lima Metropolitana en 1986, adquirimos el libro y al azar encontramos una poesia ${ }^{3}$. Al lado izquierdo se encontraba la versión quechua con el título: Chaynallatak'mi wak'an ninki; al lado derecho estaba la traducción: «Dile que he llorado...». Pero, curiosamente observamos que la versión quechua tenía tres estrofas, y la castellana cuatro. En total las líneas de la poesia en quechua tenian 15 lineas, y las del castellano, 22. Veamos:

\section{CHAYNALLATAK'MI WAKAN NINKI}

Altun pawak' siwar k'enti

altun pawak' k'ori k'enti, cartachayta apapuway yanachallayman entregaykuy.

Wak'ank'achus manañachus, llakink'achus manañachus. Wak'aykunk'a chapaychak'a, chaynallatak'mi wak'an ninki, chaynallatak'm円läkinninikeca de Letrdiéyale esta carta. Altun pawak' siwark'enti altun pawak' korik'enti caratchayta apapuway yanachallayman entregaykuy.

\section{DILEQUE HE LLORADO...}

Picaflor esmeralda

el que vuela más alto

el de las plumas doradas, picaflor esmeralda que brilla en el sol que tiemblạ en el aire hincando a las flores.

Quiero darte un encargo: mi amada está lejos, picaflor esmeralda,

No sé si llorará todavía cuando lea mi nombre, o me habrá olvidado y ya no llorará. Pero si ella se entristece dile que he llorado, dile que también Iloro recordando a la amada.
Picaflor siwar
el que vuela más alto el de las plumas doradas.

Arguedas, José Maria. Cantos y cuentos quechuas /. Lima, Municipalidad de Lima, 1986. pp. 34-35. (Munilibros 12). 
Observando ya en detalle pudimos verificar que la traducción se alejaba un tanto del original; tenía más versos, y estos contenian más expresiones que el original. Veamos nuevamente:
Altun pawak' siwar k'enti
Picaflor esmeralda
altun pawak' k'ori k'enti,
el que vuela más alto
cartachayta apapuway
el de las plumas doradas,
yanachallayman entregaykuy.
picaflor esmeralda
que brilla en el sol
que tiembla en el aire
hincando a las flores.

Es evidente que toda la estrofa castellana anterior se refiere sólo a las dos primeras líneas del quechua:

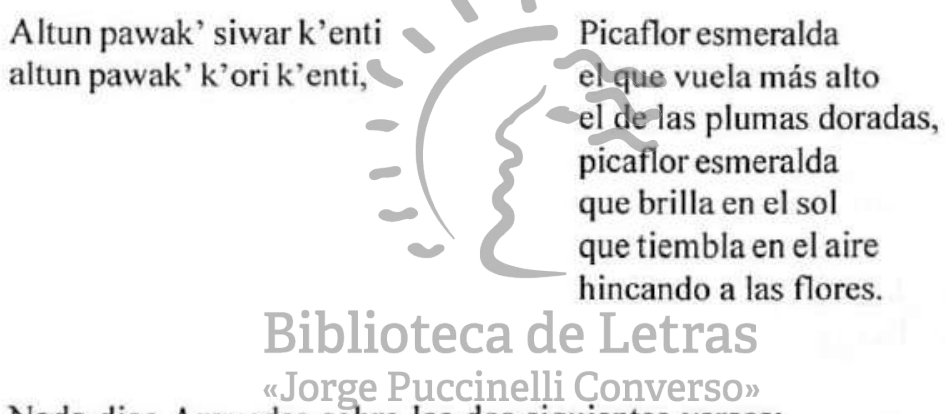

Nada dice Arguedas sobre los dos siguientes versos:

cartachayta apapuway

yanachallayman entregaykuy.

Con las otras poesías ocurría lo mismo. Algunas traducciones conservaban la cantidad de estrofas del idioma original, pero la cantidad de versos siempre era superior a la del quechua. be:

Al respecto, José María Arguedas en las primeras páginas del libro escri-

Hace tiempo que tenía el proyecto de traducir las canciones quechuas que habia oído y cantado en los pueblos de la sierra. [...] En mis lecturas no encontré 
ninguna poesía que expresara mejor mis sentimientos, que la poesia de esas canciones. ${ }^{4}$

No estamos necesariamente de acuerdo con las expresiones de Edmundo Guillén Guillén quien en una conversación personal nos manifestó que Arguedas tenía poco conocimiento del quechua; para nosotros José Maria fue un quechuahablante y como tal hablaba fluidamente el quechua de su región; aunque debemos advertir, como es de dominio general, que la variante AyacuchoChanka tiene en la actualidad sólo un $65 \%$ de vocabulario quechua y un $35 \%$ de castellano, a diferencia del quechua cusqueño que tiene 90 a $95 \%$ de quechua. Respecto a las grafias utilizadas por nuestro mestizo escritor Arguedas, debemos decir que son de libre uso. En la actualidad estas se hallan normadas de acuerdo a Ley, por la Academia Mayor de Quechua del Perú, con sede en la Capital Histórica del Perú, el Qosqo.

\section{Hipótesis}

Por lo que toca a nosotros, la hipótesis es que José María Arguedas no era traductor profesional. La traducción, es hoy, un quehacer especializado, que para el caso de la traducción poética requiere no sólo de técnicas, sino de habilidad poética, tal vez esto-ocasionó que su productor no reflejara adecuadamente el espíritu de los versos que pretendia traducir. La existencia de tratados de traductología y Teoría de la traducción en América Latina en 1939 eran nulos o escasos. Es más, posiblementeala traductologíla estaba en gestación.

\section{Verificación}

$$
\text { "Jorge Puccinelli Converso» }
$$

Justamente, es el mismo autor, quien nos ayuda a comprobar nuestra hipótesis. Con fecha, 16 de noviembre de 1959, respondió a una carta del historiador norteamericano John V. Murra, quien desde los Estados Unidos de Norteamérica le escribía: "He intentado una traducción de las frases de Avila ${ }^{5}$ qué me propone Ud.» respecto a la expresión:

...Ilactacunapipas ayllo ayllonpi...

4 Arguedas, José Maria. Cantos y cuentos quechuas I. Lima, Municipalidad de Lima, 1986, pp. 34-35. (Munilibros 12).

5 Ávila, Francisco de. "Religión en Huarochiri" publicado en 1608. El autor, sacerdote cusqueño, enviado al curato de Huarochiri, Lima, se propuso combatir de manera fundada 
El escritor peruano respondió a Murra: «Es clarisimo el significado, palabra a palabra». La expresión quiere decir:

...y también en los pueblos, de ayllo en ayllo... ${ }^{6}$

En otra carta fechada en Lima el 29 de octubre de 1966, Arguedas manifiesta:

Querido John: Me llega tu carta luego de dos dias de trabajo y de preocupación extremados a consecuencia de los errores de traducción que cometí en los dos suplementos. - [Se refiere a los suplementos de Dioses y hombres de Huarochirí (1966), y a nota de pie dice:]- Trabajé todo un día con Torero lingüista peruano, autor de numerosos ensayos sobre la lengua quechua y también del libro: El quechua y la historia social andina (Lima 1974)...- y vamos ha concluir la revisión mañana. Voy a tener que hacer imprimir de nuevo el texto íntegro de los «suplementos».

Continúa el autor y expresa:

La traducción, desgraciadamente tiene defectos. Debió haberse hecho en equipo, calmadamente, consultándonos los unos a los otros, especialmente los tres: Tú, Torero y yo.

Torero domina eBquechiua antiguo mejor que el actual ${ }^{7}[.$.$] . Es algo des-$ agradable recordar que cyando trabajaba en la traducción yo habia renunciado ya a seguir viviendo y trabaje bajo la presión de la angustia y del apresuramiento; $[$...] No creo que una sola persona pueda traducirlo con mayor aproximación posible.

Si yo hubiera sabido algo de lingüística, de paleografia y de dialectología quechua, podria haber hecho la traducción como es debido. Pero me dejé cau-

y sistemática las idolatrías prehispánicas, para ello tuvo que documentarse haciendo un registro de los mitos tradiciones. religión y demás costumbres de los indígenas de la región. Esta obra está escrita en quechua imperial.

6 Murra, V. John y Mercedes López-Baralt. Las cartas de Arguedas. Lima: Pontificia Universidad Católica del Perú, Fondo Editorial, 1996, p. 31.

7 No es cierto que el Dr. Torero dominara ningún quechua, ni antinguo ni nuevo. Como linguiista podía explicar muchas cosas como la evolución y difusión del quechua, recurriendo a una arqueolingüística; cuyos frutos como de toda ciencia experimental y de campo sólo tienen carácter de probable y no de verdadero como han querido interpretar otros. 
tivar por la parte mítica y mágica, y ahora que analizo la traducción sobre frío y con algo más de información especial sobre su importancia, me causa algo de terror y de admiración al mismo tiempo por la obra que hice. ${ }^{8}$

\section{Al final de la carta, concluye:}

Ya no hay más remedio que tomar la traducción como un aceptable borrador de traducción. Así lo he de hacer constar en el prólogo.... ${ }^{\text {? }}$

En otra carta, fechada también en Lima, el 16 de diciembre de 1966, manifiesta:

...En cuanto al Dr. Dávila, el libro siguió complicándose. Llegaron los microfilms de Madrid. Se comprobó que Loayza ${ }^{10}$ habia cambiado palabras, suprimido frases, absurdamente del texto castellano de Ávila... Y vamos a tener que volver a imprimir nuevamente toda esa parte del libro....

En cuanto a la traducción, insiste el autor nuevamente:

Debió haberse hecho en equipo, con Torero, y palabra a palabra, midiendo, pesando cada palabra. Pero acaso no se habría acabado jamás. La que ha de publicarse es, como dices, una buena base, no tan mala. [...] Si me recupero plenamente la revisaremos con Torero y la segunda edición podrá ser firme, puesta a prueba por ti ypor Zuidema, porejemplo. Estegrimer borrador dará una visión de su valor total: "Jorge Puccinelli Converso"

De las palabras de Arguedas expresadas en los párrafos citados podemos inferir las siguientes expresiones que dilucidan mejor nuestra hipótesis.

Me dejé ganar por el mito y la magia.

8 Ibid., pp. 133-134.

"Ibid., p. 136.

10 A pie de página dice: Francisco A. Loayza, editor de «Religión en Huarochiri. Crónica escrita por el Presbitero Francisco de Avila, en el año I608" en Francisco A. Loayza. Editor, Culto libre entre los Inkas. Lima: Imprenta Miranda, 1952. (Colección Los Pequeños Libros de Historia Americana. Director F.A. Loayza, T. XVII).

" Murra, V. John y Mercedes López-Baralt,. Las cartas de Arguedas. Pontificia Universidad Católica del Perú, Fondo Editorial 1996, p. 138. 
Trabajé bajo la presión de la angustia y el apresuramiento.

Debemos traducir palabra a palabra, midiendo, pesando cada palabra.

No creo que una sola persona pueda traducirlo con mayor aproximación posible.

Si yo hubiera sabido algo de lingüística, de paleografía y de dialectología quechua, podria haber hecho la traducción como es debido.

Hoy, todo traductor profesional sabe que no es posible renunciar en su totalidad a su propia cosmovisión durante el acto de traducir; pero que debe, de todos modos, introducirse en la cosmovisión del autor a quien traduce. Que no se debe trabajar bajo ninguna presión sobre todo de carácter psicológico como la angustia y menos por el apresuramiento. La traducción no se realiza palabra por palabra, sino que se traducen conceptos, ideas, sentimientos y voliciones.

Finalmente, como se ha demostrado por siglos, aún antes de contar con traductores profesionales, no sólóes impertante el dominio del idioma, de la teoría lingüística y afines, sino que es también imprescindible la formación humanística en el campo de la filosofia, la lógica, estética, la hermenéutica, la antropología, la historia de la cultura universal y la historia de la ciencia, y, por supuesto, una gran dosis de sensibilidad estética, que, claro está, no podemos negar a Arguedas.

Por el sentido de las exprèsiones, tas dimitacionesde Arguedas podemos agrupar como sigue: "Jorge Puccinelli Converso»

1. Causas de orden subjetivo:

"Me dejé ganar por el mito y la magia".

"Trabajé bajo la presión de la angustia y el apresuramiento".

2. Desconocimiento de técnicas traductológicas:

"Debemos traducir palabra a palabra, midiendo, pesando cada palabra".

"No creo que una sola persona pueda traducirlo con mayor aproximación posible".

3. Carencia de formación teórica:

"Si yo hubiera sabido algo de lingüistica, de paleografía y de dialectología quechua, podría haber hecho la traducción como es debido". 
El autor, consciente del problema motivo de nuestra exposición, había sostenido:

Insisto pues en decir que no son traducciones rigurosamente literales, son traducciones un tanto interpretativas, que quizá desagradarán un poco a los filólogos, pero que será una gran satisfacción para los que sentimos el quechua como si fuera nuestro idioma nativo.

$[\ldots]$

Me falta sólo decir que en esas versiones se encontrará, sin duda, la influencia de la parte que tendo de español, pero eso no lo podía evitar. [...]

Más tarde, otro que sienta lo indio más auténticamente que yo nos dará versiones más propias y puras. ${ }^{12}$

Conclusión

Como ya lo expresamos en la primera parte de la presente exposición, Arguedas tiene el inmenso mérito de haber emprendido el estudio de la cultura andina y haber tratado de romper sus fronteras mediante la traducción. Hoy se hace necesario que las nuevas generaciones alcancemos, a la luz de los nuevos conceptos y adelantos de la traductología, esa "objetividad" y precisión que él alcanzó parcialmente. Pensamos que antes de queqos estudios del mundo andino hayan alcanzado robjetividadicientifican transourrirá tal vez una centuria, mientras tanto, cualquier interpretación será una aproximación al mundo real. Consideramos que este articulo en modo alguno disminuye el mérito ni la figura del Hamawt'a; por el contrario, él es la piedra angular en cuyo hombro nos tenemos que parar quienes venimos atrás para construir o reconstruir el maravilloso edificio del pensamiento andino.

Termina su comentario y sostiene:

Pero si, estoy seguro de que la edición de estas canciones contribuirá a ubicar, de una vez, toda la poesia indigenista y cholista que se ha publi-

12 Arguedas, José Maria. Op. cit., p. 31. 
cado hasta hoy. Y enseñará la posibilidad de una poesia de tema y de espiritu indigena. ${ }^{13}$

este arte popular podrá ser el fermento, la raiz primaria de una gran producción nacional en todos los aspectos del arte. ${ }^{14}$

Presentamos a continuación una traducción alternativa de los versos en cuestión, y pretendemos que constituyan un mayor acercamiento al espíritu y sensibilidad del mundo quechua andino. La versión quechua está normalizada por los autores del artículo de acuerdo a las directivas emanadas de la Academia Mayor de Quechua del Perú.

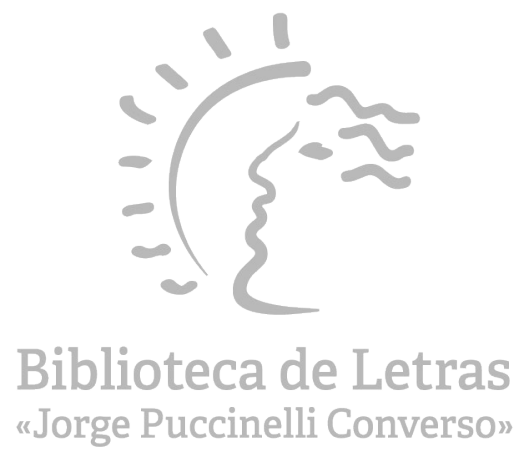

17 Ibíd. p. 31.

14 Ibid, p. 31. 
CHAYNALLATAK'MI WAKAN NINKI

Recopilación: José Maria Arguedas

Altun pawak' siwar k'enti

altun pawak' k'ori k'enti, cartachayta apapuway

yanachallayman entregaykuy.
DILE QUE HE LLORADO...

Traducción: José María

Arguedas

Picaflor esmeralda

el que vuela más alto

el de las plumas doradas, picaflor esmeralda

que brilla en el sol

que tiembla en el aire

hincando a las flores.

Quiero darte un encargo:

mi amada está lejos, picaflor esmeralda, llévale esta carta.

Wak'ank'achus manañachus, Ilakink'achus manañachus.

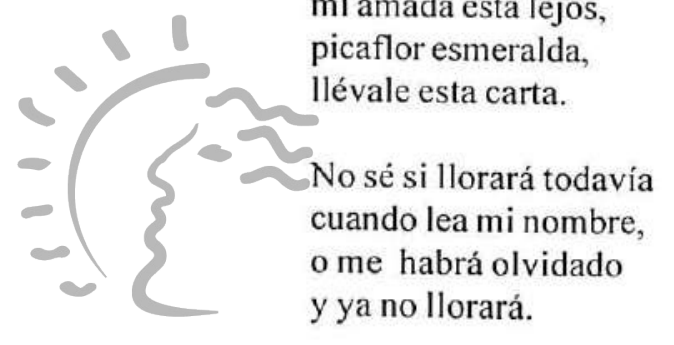

Wak'aykunk'a chapaychak chaynallatak'mi wak'aningki,uccinelli Convaleque he llorado, chaynallatak'mi Ilakin ninki.

Altun pawak' siwar k'enti

altun pawak' korik'enti

cartachayta apapuway

yanachallaman entregaykuy. dile que también lloro recordando a la amada.

Picaflor siwar

el que vuela más alto el de las plumas doradas. 
CHHAYNALLATAQMI WAQAN NINKI.

Normalización: Mario Mejia

Hanan phawaq siwar q'enti hanan phawaq qori q'enti kartachayta apapuway yanachayman qoykapuway
DILE QUE TAMBIEN LLORA ASI
Picaflor tornasolado

ora verde, ora dorado sobre tus alas doradas

lleva esta cairta a mi amada.

Wanqanqachus

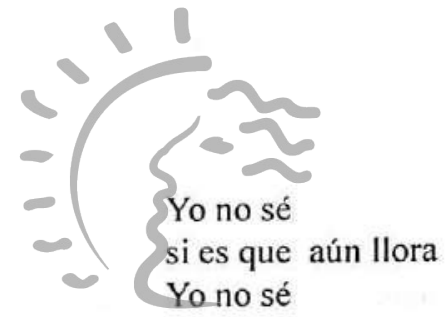

llakinqachus si es que aún sufre. manañachus.

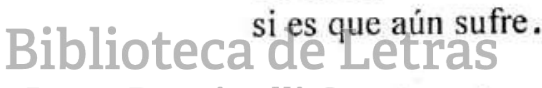

Waqaykunqa chaypachäqarge Puccinsklịlorandoestáa raudales chhaynallataqmi waqan ninki chhaynallataqmi llakin ninki.

Hanan phawaq siwar q'enti hanan phawaq qori q'enti kartachayta apapuway yanachayman qoykapuway. dile que así también Iloro dile que así también sufro.

Picaflor tornasolado ora verde, ora dorado sobre tus alas doradas lleva esta carta a mi amada. 
CILILI WAYTA

Recopilación: José María

Arguedas.

Intillay, killallay

ama sak'ewaychu,

karurak'mi rinay

tutayallaymanmi.

Sumak' siwark'enti, ama jarkawaychu, mamallaysi maskawan uñan chinkachik' urpi jina.
CILILI, HERMOSA FLOR

Traducción: José María Arguedas.

¡Oh Sol, oh Luna, alumbrad mi camino!

No bajes tan temprano Sol, alumbra todavía.

Tarda un poco Luna , es lejos mi destino, tengo miedo a la sombra.

Picaflor siwar, oculta tus alas doradas, no me atajes picaflor siwar, es largo mi camino.

Como paloma que ha perdido a su polluelo está llorando mi madre; no meatajes picaflor siwar.

Cilili, cilili wayta, k'awachkankim kay vidayta mayu jina wak'ask'ayta 10

wayra jina k'aparispa il lio como el fío como el viento, eilit, hermosa flor. "Jorge Puccinelli. Converso" 
SILILI WAYTA

Normalización: Mario Mejía

Intillay killallay

ama saqewaychu,

karuraqmi purinay

tutayawanmanmi.

Sumaq siwar q'enti, ama hark'awaychu, mamallaysi maskhawan uñan chinkachikuq urpi hina.
FLOR DE SILILI

Traducción: Mario Mejia y César

Saldaña

Mi solcito, mi lunita

les ruego que no me dejen

mi destino es muy lejano

y podría anochecerme.

Picaflor tornasonaldo

no me impidas que yo vuelva

dicen que mi madre me busca

cual paloma a su polluelo.
Silili, silili wayta, qhawaskankin kay vidayta mayu hina waqasqayta wayra hina qaparisqayta.
Silili, hermosa flor,

testigetú de mi amor

Illorando a mares estoy

gimiendo cual viento voy.

\section{ISCHU KAÑASKAY}

\section{Biblioteca de Letras}

Recopilación: José María Arguedas

Ork'opi ischu kañask'ay,

k'asapi ischu kañask'ay

¡Jinallarak'chus rupachkan

jinallarak'chus raurachkan!

Jinalla raurariptink'a, jinalla rupariptink'a, ¡Warma wek'echaykiwan challaykuy!

¡Warma wek'echaykiwan

tasnuykuy!

\section{EL FUEGO QUE HE PRENDIDO}

Traducción: José María Arguedas

El fuego que he prendido en la montaña

el ischu que encendí en la cumbre estará llameando

estará ardiendo.

¡Oh mira si aún llamea la montaña!

Y si hay fuego ¡anda niña!

Con tus lágrimas puras

apaga el fuego;

llora sobre el incendio

y tórnalo en cenizas con tus lágrimas

puras. 
ICCHU KANASQAY

Normalización: Mario Mejía

Orqopi icchu kanasqay, q'asapi ichhu kanasqay hinallaraqchus ruphashkan hinallaraqchus rawrashkan.
ICHHU QUE QUEME

Traducción: Mario Mejía y César

Saldaña

La paja que en el cerro quemé, la paja que en el abra prendí, ¿estará aún ardiendo? ¿estará aún quemando?

Hinalla yawrashaqtinqa hinalla ruphasaqtinqa warma weqechaykiwan ch'allaykuy, warma weqechaykiwan thasnuykuy.
Si estuviera aún ardiendo, si estuviera aún quemando, rocíalo con tu llanto con tus lágrimas de joven, rocíala con tus lágrimas con tus lágrimas de joven.
AMAYA TARILLACHUNCHU

Recopilación: José María Arguedas

Ork'opi wikuña, k'asapi taruka tapurikullask'ayki: kainintachus pasallark'ablioteca dedeciatmesispasó por aquí la ingrata yanallan sak'erik' urpiJorge Puccinelli Converso» la paloma que dejó su nido que olvidó a su amado.

Kayk'aya sak'erk'ullawan, kayk'aya dejar k'ullawan ñawillay junta wek'entinta sonk'ollay junta llakintinta.

Yanan sak'erik'urpi !amaya tarillachunchu! wayllay ischupa sulluntapas yakunayaptin suk'uykunampak.'
Vicuña de los cerros, taruka de los montes, venid a ver cómo lloran mis ojos; así me dejó, con el los ojos llorando, así me dejó, con el corazón herido.

¡Oh, que tenga sed en el camino! y que no encuentre ni la escarcha en los pajonales que no encuentre ni el rocio en las yerbas.

¿Que tenga sed en todos los caminos la paloma que olvidó a su amado! 
Normalización: Mario Mejia Waman

Orqopi wik'uña, q'asapi taruka tapurikullasqayki:

kaynintachus pasallarqan yanallan saqeriq urpi.

Kayqayá saqerqollawan, kayqayá dejarqollawan ñawillay hunt'a weqentinta, sonqollay hunt'a llakintinta.

\section{QUENOENCUENTRE}

Traduc: Mario Mejia y César Saldaña

Venado del abra, vicuña de la montaña, quiero hacer una pregunta ¿vieron pasar la paloma que ha abandonado su nido?

Miren, pues, que me ha dejado miren, pues, me ha abandonado mis ojos llenos de llanto y el corazón de quebranto
Yanan saqerikuq urpi, Amaya tarillachunchu! wayllay ichhupa chhullantapas yakunayaqtin soq'oykunanpaq
Que la que deja a su amado ¡que ni siquiera halle rocío! en las pajuelas del ichhu, para que aplaque su sed!

Recopilación: José MarỉaAirgiedasa do Traduccions José Maria Arguedas.

Alkunchallay wamanchallay

alaykipi apakuway

alaypiki apawaspa

ñanchallaman churaykuway.

Alkunchallay wamanchallay kay ork'onpim chinkark'uni, alaykipi apaykuway ñanchallaman churaykuway.

Chaymantak'a ripusak'mi, chaymantak'a pasasak'mi viagueruwan tupaykuspa wamanguinuwan tak'rukuspay
Gavilan del cielo, halcón de las alturas, baja un rato, me he perdido en estos montes, llévame en tus alas hasta el camino.

Me he perdido en estos montes, gavilán de las altura Yo sólo quiero que me lleves al camino, baja un rato, halcón del cielo.

Déjame en el camino, halcón.

De allí me iré con los viajeros, con la tropa de los wamangas, !gavilan, baja un rato! 
Wamanchallay, wamanchallay Halconcito, mi halconcito raphraykipi apakuway, elévame con tus alas, raphraykipi apawaspa ñanchallaman churaykuway. y llevándome en tus alas guíame a mi caminito.

Wamanchallay, wamanchallay kay orqopin chinkarqoni, raphraykipi apaykuway ñanchallaman churaykuway.

Halconcito mi halconcito en el cerro me he perdido, llévame pues en tus alas guíame a mi caminito

Chaymantaqa ripusaqmi, Chaymantaqa pasaqmi, viajerowan tupaykuspa, wamanguinuwan taqrukuspay.

Una vezen mi camino, desde alli podré marcharme, de allí podré acompañarme, junto con un huamanguino.

\section{ORQOKUNAPI WAYLLAR ICHHUPAS TAMBIENLOS ICHHUS DELANDE}

Normalización: Mario Mejı́a Wamannelli Gpaduc: Mario Mejia y César Saldaña

Orqokunapi wayllar ichhupas para chayaqtin chhullaykachansi, chaynan noqapas waqallashkani runaq wasinpi rikuykukuspay runaq llaqtanpi qhawaykukuspay.

Orqokunapi wayllar icchupas wayra muyuqtin k'umuykachansi, chhaynan noqapas k'umuykachani runaq wasinpi rikuykukuspay runaq llaqtanpi qhawaykukuspay.
Dicen que los ichhus del Ande cubiertos son de rocío, así yo llorando estoy en hogar que no es el mío, en tierra que no es la mía.

Dicen que los ichhus del Ande cuando sopla el viento agachan, la cabeza igual que yo, en casa que no es la mía en tierra que no es la mía. 
ORK'OKUNAPI WAYLLAR ISCHUPAS

Recopilación: José Maria Arguedas

Ork'okunapi wayllar ischupas para chayaptin sullaykachansi, chaynam ñok'apas wak'allachkani runapa wasimpi rikuykukuspay runapa llak'tampi k'awaykukuspay.

\section{EL ISCHU ESTA LLORANDO}

Traducción: José Maria Arguedas

Cae la lluvia en las lomadas queda la escarcha en los pajonales. Pasa la lluvia, sacude el viento, del ischu gotea el agua gotea el agua limpia.

!El ischu está llorando!

!Ay, cómo lloran los ojos en el pueblo ajeno!

Lloran los ojos como llora el ischu cuando pasa la Iluvia y sopla el viento.

Cuando sopla el viento el ischu se agacha, el ischu alto de las lomadas se agacha cuando sopla el viento.

wayra muyuptin kumuykachansi, chaynam ñok'apas kumuykachani runapa wasimpi rikuykukuspay runapa llak'tampi k'awaykukuspay.
!Ay, como se dobla el corazón en pueblo ajeno!

Como el ischu alto cuando sopla el viento.

\section{Biblioteca de Letras «Jorge Puccinelli Converso»}

